\section{Anthracycline-related cardiotoxicity: risk factors and therapeutic options in childhood cancers}

\author{
NADIA PUMA • ANTONIO RUGGIERO • VITA RIDOLA • PALMA \\ MAURIZI • ILARIA LAZZARESCHI • GIORGIO ATTINÀ • STEFANO \\ MASTRANGELO • GABRIELLA DE ROSA • RICCARDO RICCARDI
}

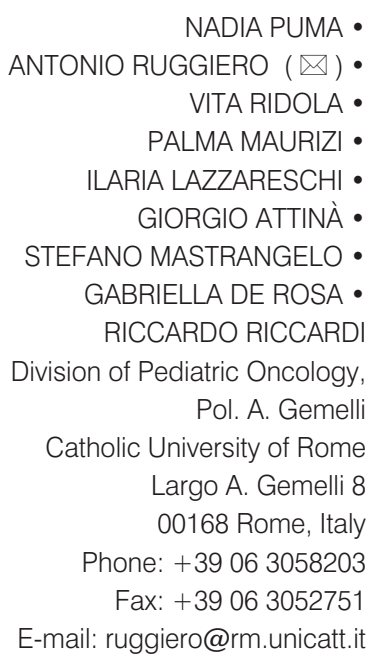

NADIA PUMA • ANTONIO RUGGIERO $(\square) \bullet$ VITA RIDOLA • PALMA MAURIZI • ILARIA LAZZARESCHI • GIORGIO ATTINÀ • STEFANO MASTRANGELO • GABRIELLA DE ROSA • RICCARDO RICCARDI Division of Pediatric Oncology, Pol. A. Gemelli Catholic University of Rome Largo A. Gemelli 8 00168 Rome, Italy Phone: + 39063058203

Fax: +3906 3052751

E-mail: ruggiero@rm.unicatt.it

\begin{abstract}
Anthracyclines play an important role in chemotherapeutic regimens for a wide spectrum of childhood tumors, but they can cause cytotoxic damage to cardiac cells, especially in combination with radiotherapy. Furthermore, cardiotoxicity increases with the cumulative dose and may lead to congestive heart failure and cardiomyopathy. Other factors, including age, pre-existing cardiac disease, length of follow-up, gender, route of administration, concomitant exposure to some chemotherapeutic drugs, trisomy 21 and black race, play a role in increasing the risk of cardiac dysfunction. The prevention of anthracycline-induced cardiotoxicity is mandatory as children are expected to survive for decades after being cured of their cancer. The purpose of this work is to point out the major risk factors of cardiotoxicity in children and to summarize some strategies to limit or prevent this complication and to treat the development of acute heart failure.
\end{abstract}

Keywords: anthracycline, cardiotoxicity, childhood cancer, risk factors, prevention, acute heart failure

\section{Introduction}

Anthracyclines (daunomycin, doxorubicin, epirubicin, idarubicin) are among the most effective antineoplastic drugs and have gained widespread use in the treatment of numerous solid tumors and hematological malignancies.

These drugs belong to the class of antineoplastic antibiotics which interfere with cell replication, by acting on DNA at several levels showing an effect in every phase of the cell cycle.

Doxorubicin and daunomycin (figure 1) are the more commonly used anthracyclines in clinical practice; they are administered only by intravenous infusion and metabolized by the liver. Their main route of excretion is through bile, while urinary elimination accounts for approximately $1 / 6$ of the total amount. Although anthracyclines exhibit a range of toxic effects including myelosuppression, mucositis and hair loss, cardiotoxicity may be permanent and progressive.<smiles>COc1cccc2c1C(=O)c1c(O)c3c(c(O)c1C2=O)CC(O)(C(=O)CO)CC3OC1CC(N)C(O)C(C)O1</smiles>

Doxorubicin
Therefore, the oncologist should take into account the risk of cardiac toxicity due to anthracyclines as a potential cause of severe morbidity in survivors of childhood cancers, as children will have a chance of long-term survival once cured from cancer. We reviewed<smiles>COc1cccc2c1C(=O)c1c(O)c3c(c(O)c1C2=O)CC(O)(C(C)=O)CC3OC1CCC(N)C(O)C(C)O1</smiles>

Daunomycin

Figure 1. Structures of the anthracyclines currently used. 
the spectrum of clinical presentations of anthracycline-induced toxicity, focusing on acute heart failure.

\section{Mechanism of cardiotoxicity}

Three mechanisms are involved in the cytotoxic action of anthracyclines:

1. high affinity binding to DNA, via nucleic intercalation, which causes inhibition of DNA and RNA synthesis and cleavage of DNA strands by alterations of topoisomerase II

2. binding to the cell membrane which alters its normal fluidity and transport of ions

3. semiquinonic and oxygen free radical production, via reduction enzymatic reaction.

Despite the wide use of anthracyclines, their cardiotoxic mechanism is still not completely understood. The most common hypothesis is the formation of free radicals and superoxides. (1-4) Cardiac cells are more susceptible to free radical damage because of their highly oxidative metabolism and relatively poor antioxidant defences. (5) This mechanism was originally proposed as the basis for anthracyclinesrelated cardiotoxicity (6), but some additional variables are involved, such as the interaction of the anthracyclines with iron.

\section{Epidemiology}

In the literature, there is wide variation in the reported frequency of both clinical and subclinical cardiotoxicity.

In children, early onset cardiotoxicity seems to occur less frequently than late cardiac damage. There is a relatively low rate of early clinical cardiotoxicity, since only $1.6 \%$ of all children treated with anthracyclines have cardiotoxic effects. (7)

The prevalence of late subclinical cardiac damage has been reported to be more than $57 \%$ at a median of 6.4 years after treatment among survivors of childhood cancers (8) and the incidence of clinical heart failure as high as $16 \%, 0.9$ to 4.8 years after treatment. (9) Differences in study population, treatment protocols, and duration of follow-up could account for this wide variability.

The risk of cardiotoxic effects occurring about 15 years after treatment with a mean cumulative dose of anthracyclines of $300 \mathrm{mg} / \mathrm{m}^{2}$ is estimated to be approximately $5 \%$. (10)

Risk of mortality from cardiac-related events is 8 times higher for long term survivors than for the normal population. $(11,12)$

\section{Risk factors for anthracycline-induced cardiotoxicity}

Several risk factors for anthracyclineinduced cardiotoxicity appear to be involved in the development of clinical and subclinical cardiotoxic effects. Patients should be screened for risk factors and an attempt to modify them should be made.

The most important risk factors for anthracycline-induced cardiotoxicity are summarized in table 1.

\begin{tabular}{ll} 
RISK FACTORS EFFECTS \\
\hline
\end{tabular}

\begin{tabular}{|c|c|}
\hline Abnormal cardiac function & $\begin{array}{l}\text { Higher risk for chronic cardiac } \\
\text { dysfunction }\end{array}$ \\
\hline Cumulative dose & $\begin{array}{l}\text { The most significant risk factor for } \\
\text { cardiac dysfunction }\end{array}$ \\
\hline Age & $\begin{array}{l}\text { Younger age predisposes to greater } \\
\text { cardiotoxicity }\end{array}$ \\
\hline Lenght of infusion & $\begin{array}{l}\text { Infusions do not differ from bolus } \\
\text { modality in children. }\end{array}$ \\
\hline Sex & Females are more vulnerable \\
\hline Irradiation & $\begin{array}{l}\text { Concomitant mantle irradiation is a } \\
\text { predisposing factor }\end{array}$ \\
\hline $\begin{array}{l}\text { Additional treatment } \\
\text { (cyclophosphamide, bleomycin, } \\
\text { vincristine or mitoxantrone) }\end{array}$ & Predispose to cardiotoxicity \\
\hline Black race & Higher risk of early clinical cardiotoxicity \\
\hline Trisomy 21 & Higher risk of early clinical cardiotoxicity \\
\hline Length of follow-up & $\begin{array}{l}\text { A longer follow-up increases the risk of } \\
\text { cardiotoxicity }\end{array}$ \\
\hline
\end{tabular}

Total cumulative dose of anthracyclines is the most significant risk factor for cardiac dysfunction. (13) The maximum cumulative dosage linked to minimal cardiotoxicity varies among the different anthracyclines and a safe dose, not resulting in late cardiac injury, has not been established.

The usual dosage of doxorubicin is 60$75 \mathrm{mg} / \mathrm{mq}$, every 3 weeks. (14) Above a cumulative dose of $450-500 \mathrm{mg} / \mathrm{mq}$, cardiomyopathy and congestive heart failure occur frequently.

Younger age predisposes to greater cardiotoxicity. (15) Children seem to be more sensitive to the cardiac adverse effects of the anthracyclines. However, in children mechanisms leading to late cardiotoxicity differ from those causing acute cardiotoxicity in adults $(13,16)$ and anthracycline infusions were used in order to lower peak drug concentration, reducing cardiotoxicity.

Hypertension and any significant preexisting cardiac disease increase the

\section{Table 1. Risk factors for anthracyclines-related cardiotoxicity.}


risk of clinically apparent cardiac disease. (17)

Females are more vulnerable than males for comparable doses. (7)

Concomitant mantle irradiation is a predisposing factor, but it is not clear whether it is additive or synergistic to treatment with anthracyclines. (15) Cardiac radiation exposure clearly increases the sensitivity of the heart to anthracyclines. Concomitant exposure to cyclophosphamide, bleomycin, vincristine, amsacrine or mitoxantrone may predispose to cardiotoxicity. (7)

Blackrace (7) and trisomy 21 (7) increase the risk of early cardiotoxicity.

An increased incidence of cardiac failure and severe cardiac abnormalities more frequently occur with longer follow-up. (15)

\section{Clinical presentation}

The clinical presentation of anthracycline-induced toxicity can be divided into:

- acute or subacute: heart damage that develops immediately after the infusion of the drug or within a week of therapy - early onset chronic progressive cardiotoxicity: a depression of myocardial function which occurs during the treatment or within the first year after treatment

- late onset chronic progressive cardiotoxicity: this occurs at least 1 year after the end of treatment.

Clinically significant cardiotoxicity during or in the first year following initial chemotherapy is rare, occurring in less than $1 \%$ of children. (13) Instead, late subclinical abnormalities of left ventricular structure and function in long-term survivors of childhood cancer are common. $(13,16,18-23)$ Late onset effects can occur up to 20 years after completion of anthracycline therapy. (19)

Early cardiotoxicity is presumably related to myocyte damage or death resulting in depressed left ventricular contractility $(13,16,24)$; while late cardiotoxicity is due to depressed contractility and thin left ventricular wall resulting in increased wall stress and decreased left ventricular systolic function. $(13,16,24)$

\section{Acute heart failure related to anthracycline induced cardiotoxicity}

Acute heart failure in children receiving anthracyclines can occur within one week of drug infusion, thus without the presence of chronic cardiac damage, or as acute decompensated heart failure in the setting of established anthracycline-induced cardiomyopathy. The second scenario is more common and develops when underlying compensatory mechanisms that sustain normal cardiac function in the setting of chronic heart failure, are exhausted.

Acute changes during anthracycline infusion range from minor electrocardiographic abnormalities (non-specific ST-T changes and QT prolongation) to fatal ventricular arrhythmias. Echocardiography can reveal LV systolic dysfunction that is usually transient, but a few patients may experience acute and potentially fatal congestive heart failure while on therapy. (25) In its more extreme form, this acute injury can include a pericarditis-myocarditis syndrome with onset of fever, pericarditis and congestive heart failure. This syndrome can occur at low cumulative doses of doxorubicin and can have a fatal outcome. (26)

The Ross Classification (27) was developed for grading heart failure in infants and younger children (Grade I: asymptomatic. Grade II: mild tachypnea or diaphoresis with feeding in infants; dyspnea on exertion in older children. Grade III: marked tachypnea or diaphoresis with feeding in infants; prolonged feeding times with growth failure due to heart failure; in older children, marked dyspnea on exertion. Grade IV: symptoms such as tachypnea, retractions, grunting, or diaphoresis at rest).

Breathlessness, a cardinal manifestation of left ventricular failure, may present, in order of severity, as:

- exertional dyspnea (as left ventricular failure progresses, the intensity of exercise resulting in breathlessness declines progressively)

- orthopnea (dyspnoea that develops in the recumbent position and is relieved by elevation of the head with pillows)
- paroxysmal nocturnal dyspnoea (feeling of severe anxiety and suffocation and gasps for breath)

- acute pulmonary edema (is considered a clinical emergency and occurs as initial presentation of heart failure or as an aggravating condition of heart failure. Presents as extreme breathlessness that develops suddenly, the respiratory rate is elevated, the alae nasi are dilated and there is inspiratory retraction of the intercostal space and use of accessory muscles of respiration; the skin is usually cold and cyanotic reflecting low cardiac output. On auscultation there are many lung sounds with ronchi, wheezes and fine crepitant rales that appear at first over the lung bases but then extend upward to the apices; cardiac auscultation is difficult because of the respiratory sounds).

Cardiomegaly occurs in patients with chronic systolic heart failure. Also, protodiastolic sounds (gallop sounds) are audible in patients with heart failure. In mild right-sided heart failure, the jugular venous pressure may be normal at rest but rises to abnormal levels with compression of the right upper quadrant (hepatojugular reflux).

The liver often enlarges before overt edema develops and it may remain so even after other symptoms of rightsided heart failure have disappeared. Although a cardinal manifestation of heart failure, edema does not correlate well with the level of systemic venous pressure. Edema in heart failure is usually symmetrical and pitting and generally occurs in the dependent portions of the body. Late in the course of heart failure, edema becomes massive and generalized (anasarca) and weight gain associated with anasarca correlates with attenuation of electrocardiographic voltage.

In severe heart failure prolonged sodium restriction coupled with intensive diuretic therapy and the inability to excrete free water, may lead to dilutional hyponatremia. Serum potassium levels are usually normal, although prolonged administration of kaliuretic diuretics may result in hypokalemia. Other common electrolyte abnormalities include 
hypophosphatemia, hypomagnesemia and hyperuricemia.

Proteinuria and a high urine specific gravity are common findings in heart failure.

Arrhythmias likely to require acute treatment in patients with heart failure include atrial tachyarrhythmias with a rapid ventricular rate, paroxysmal supraventricular tachycardia, junctional ectopic tachycardia and sustained ventricular tachycardia. Synchronized cardioversion/defibrillation should be considered for treatment of either supraventricular or ventricular arrhythmias if hemodynamic collapse is imminent. $(28,29)$ Management of episodes of acute heart failure includes therapy with diuretics, support of pump function with positive inotropic agents and/or, if extremely severe, with mechanical devices to the extent necessary to provide adequate perfusion of critical organs.

Mechanical circulatory support has become an important addition to the treatment armamentarium for the infant or child with acutely decompensated heart failure and low cardiac output unresponsive to pharmacologic manoeuvres. Mechanical support maintains end-organ function and reduces myocardial oxygen requirements during a critical period of recovery from a cardiac insult.

\section{Conclusion}

Anthracycline-induced cardiotoxicity is a limiting factor in the use of anthracyclines, especially when predisposing factors are present. Therefore, the presence of risk factors should be investigated before starting therapy. Indeed, prompt recognition of modifiable risk factors can help reduce cardiac damage.

Another goal is to monitor the patient for the rapid development of initial signs of cardiac damage. Serial monitoring (echocardiogram, electrocardiogram and holter) of cardiac function in children receiving anthracyclines allows early identification of heart damage. The monitoring schedule varies between protocols. Frequency and timing of tests depends on the child's age at time of exposure, cumulative dose of anthracyclines, chest irradiation, presence of cardiac abnormalities, symptoms, and other stressors. (30) Generally, all survivors treated with cardiotoxic therapy should be screened, symptomatic or not. The protocol for the assessment of cardiac function in these patients should be based on a multi-modality approach. Acute heart failure is a dangerous complication that can develop either as acute cardiotoxicity, within one week of drug infusion, or as acute decompensated chronic heart failure, often requiring therapy with diuretics and support of pump function with positive inotropic agents.

\section{REFERENCES}

1. Alderton PM, Gross J, Green MD. Comparative study of doxorubicin, mitoxantrone and epirubicin in combination with ICRF-187 (ADR-529) in a chronic cardiotoxicity animal model. Canc Res 1992;52:194-201.

2. Rossi F, Fillippelli W, Russo S, Fillippellie A, Berrino L. Cardiotoxicity of doxorubicin: effects of drugs inhibiting the release of vasoactive substances. Pharm Tox 1994;75:99-107.

3. Vasquez-Vivar J, Martasek P, Hogg N, Masters BS, Pritchard KA Jr, Kalyanaraman B. Endothelial nitric oxide synthase-dependent superoxide generation from adriamycin. Biochem 1997;36:11293-97.

4. Rajagopalan S, Politi PM, Sinha BK, Myers CE. Adriamycin induced free radical formation in the perfused rat heart: implications for cardiotoxicity. Cancer Res 1988;48:4766-9.

5. Doroshow JH, Locker GY, Myers, CE. Enzymatic defenses of the mouse heart against reactive oxygen metabolites. J Clin Invest 1980;65:128-35.

6. Myers CE, McGuire WP, Liss RH, Ifrim I, Grotzinger K, Young RC. Adriamycin: the role of lipid peroxidation in cardiac toxicity and tumour responce. Science 1977;197:165-8.

7. Krischer JP, Epstein S, Cuthbertson DD, Goorin AM, Epstein ML, Lipshultz SE. Clinical cardiotoxicity following anthracycline treatment for childhood cancer: the Paediatric Oncology Group Experience. J Clin Oncol 1997;15:544-52.

8. Kremer LC, Van der Pal HJ, Offringa M, Van Dalen EC, Voute PA. Frequency and risk factors of subclinical cardiotoxicity after anthracycline therapy in children: a systematic review. Ann Oncol 2002;13:819-29.

9. Kremer LC, van Dalen EC, Offringa M, Voute PA. Frequency and risk factors of anthracycline-induced clinical heart failure in children: a systematic review. Ann Oncol 2002;13:503-12.

10. Kremer LC, Caron HM. Anthracycline cardiotoxicity in children. N Engl J Med 2004; 351:120-1.

11. Green DM, Grigoriev YA, Nan B, Takashima JR, Norkool PA, D'Angio GJ, et al, editors. Congestive heart failure after treatment for Wilms' tumour: a report from National Wilms' Tumour Study Group. J Clin Oncol 2001;19:1926-34.

12. Mertens AC, Yasui Y, Neglia JP, Potter JD, Nesbit ME Jr, Ruccione K, et al, editors. Late mortality experience in five-year survivors of childhood and adolecent cancer: the Childhood Cancer Survivor Study. J Clin Oncol 2001;19:3163-72. 
13. Lipshultz SE, Colan SD, Gelber RD, Perez-Atayde AR, Sallan SE, Sanders SP. Late cardiac effects of doxorubicin therapy for acute lymphoblastic leukaemia in childhood. N Engl J Med 1991;324:808-15.

14. Von Hoff DD, Layard MW, Basa P, Davis HL Jr, Von Hoff AL, Rozencweig M, et al, editors. Risk factors for doxorubicin-induced congestive heart failure. Ann Intern Med 1979;91:710-7.

15. Pein F, Sakiroglu O, Dahan M, Lebidois J, Merlet P, Shamsaldin A, et al, editors. Cardiac abnormalities 15 years and more after adryamicin therapy in 229 childhood survivors of a solid tumour at the Institute Gustave Roussy. Brit J Cancer 2004;91:37-44.

16. Lipshultz SE, Lipsitz SR, Mone SM, Goorin AM, Sallan SE, Sanders SP, et al, editors. Female sex and higher drug dose as risk factors for late cardiotoxic effects of doxorubicin therapy for childhood cancer. N Engl J Med 1995;332:1738-43.

17. Lipshultz SE, Sallan SE. Cardiovascular abnormalities in long-term survivors of childhood malignancy. J Clin Oncol 1993;11:1199-203.

18. Yeung ST, Yoong C, Spink J, Galbraith A, Smith PJ. Functional myocardial impairment in children treated with anthracyclines for cancer. Lancet 1991;337:816-8.

19. Steinherz LJ, Steinherz PG, Tan CT, Heller G, Murphy ML. Cardiac toxicity 4 to 20 years after completing anthracycline therapy. JAMA 1991;266:1672-7.

20. Larsen RL, Jakacki RI, Vetter VL, Meadows AT, Silber JH, Barber G. Electrocardiographic changes and arrhythmias after cancer therapy in children and young adults. Am J Cardiol 1992;70:73-7.

21. Leandro J, Dyck J, Poppe D, Shore R, Airhart C, Greenberg M, et al, editors. Cardiac dysfunction late after cardiotoxic therapy for childhood cancer. Am J Cardiol 1994;74:1152-6.

22. Sorensen K, Levitt G, Sebag-Montefiore D, Bull C, Sullivan I. Cardiac function in Wilms' tumour survivors. J Clin Oncol 1995;13:1546-56

23. De Wolf $D$, Suys B, Maurus R, Benoit $Y$, Verhaaren $H$, Matthijs $D$, et al, editors. Dobutamine stress echocardiography in the evaluation of late anthracycline cardiotoxicity in childhood cancer survivors. Pediatr Res 1996;39:504-12.

24. Lipshultz SE, Colan SD. The use of echocardiography and Holter monitoring in the assessment of anthracycline-treated patients. In: Bricker JT, Green DM, DAngeo G, editors. Long Term Complications of Treatment of Children and Adolescents for Cancer. Philadelphia (PA): Wiley-Liss; 1993. pp. 45-62.

25. Adams MJ, Lipshultz SE. Pathophysiology of anthracyclines- and radiation-associated cardioyopathies: implications for screening and prevention. Pediatr Blood Cancer 2005;44:600-6.

26. Bristow MR, Thompson PD, Martin RP. Early anthracycline cardiotoxicity. Am J Med 1978;65:823-32.

27. Ross RD, Daniels SR, Schwartz DC, Hannon DW, Shukla R, Kaplan S. Plasma norepinephrine levels in infants and children with congestive heart failure. Am J Cardiol 1987;59:911-4.

28. Bossaert L, Van Hoeyweghen R. Bystander cardiopulmonary resuscitation (CPR) in out-of-hospital cardiac arrest. The Cerebral Resuscitation Study Group. Resuscitation 1989;17 Suppl:S55- 69; discussion S199-206.

29. Nichol G, Stiell IG, Laupacis A, Pham B, De Maio VJ, Wells GA. A cumulative meta-analysis of the effectiveness of defibrillator-capable emergency medical services for victims of out-of-hospital cardiac arrest. Ann Emerg Med 1999;34:517-25.

30. Landier W, Bhatia S, Eshelman DA, Forte KJ, Sweeney T, Hester AL, et al, editors. Development of risk-based guidelines for paediatric cancer survivors: the Childrenžs Oncology Group Long-Term Follow-Up Guidelines from the Childrenžs Oncology Group Late Effects Committee and Nursing Discipline. J Clin Oncol 2004;22:4979-90. 